\title{
ORIGINAL ARTICLE Ejaculation and sperm characteristics in men with cauda equina and conus medullaris syndromes
}

\author{
N Hadiji ${ }^{1}$, R Mieusset ${ }^{2}$, JG Previnaire ${ }^{3}$, E Castel-Lacanal $^{2}$ and JM Soler ${ }^{1}$
}

Study design: Retrospective.

Objectives: The objective of the study was to describe the type of ejaculation in patients with cauda equina (CE) and conus medullaris (CM) lesions, and to analyse sperm quality.

Setting: France.

Methods: One hundred sixty-six patients with CE and CM lesions were included. Diagnosis was based on clinical (no motor responses, sensation or sacral reflexes) and urodynamic assessments (no detrusor activity). Vibromassage (VM) was used to induce ejaculation according to the recommendations for patients with spinal cord injury. If ejaculation did not occur, oral midodrine was administered in progressive doses. Retrograde ejaculation was systematically sought. Sperm parameters were analysed according to World Health Organisation recommendations (2010).

Results: Eighty-nine patients were included. Eleven ejaculated on the first VM trial (four anterograde (AE), six retrograde (RE) and one antero-retrograde (ARE)). Five patients continued trials of VM alone, two of whom ejaculated following a mean 1.9 trials (one RE, one ARE). Twenty-six patients underwent trials of VM+ midodrine, 18 of whom ejaculated following a mean 4.4 trials with a mean dose of $22.5 \mathrm{~g}$ of midodrine ( $2 \mathrm{AE}, 13 \mathrm{RE}$ and $5 \mathrm{ARE}$ ). Fifty-three ejaculates from 26 patients were analysed. Sperm concentration was low in $90.6 \%$ of samples; total necrospermia was found in $65 \%$ and asthenospermia in $95 \%$ of samples.

Conclusion: Ejaculation is difficult to induce using VM in patients with CE and CM lesions, and requires high doses of midodrine. Sperm counts were generally low, and asthenospermia and necrospermia were found in the majority of specimens. Cryopreservation of sperm should be systematic in case of medically assisted procreation.

Spinal Cord (2017) 55, 612-617; doi:10.1038/sc.2017.5; published online 14 February 2017

\section{INTRODUCTION}

In patients with spinal cord injury (SCI) with a spastic perineum (supra-sacral lesions), sperm parameters change as soon as the second week post lesion-onset. ${ }^{1}$ Motility and vitality are particularly affected; however, the changes do not worsen with time. ${ }^{1,2}$ Sperm count remains normal ${ }^{3,4}$ according to the norms determined by the World Health Organisation (WHO).

The majority of studies have focused on patients with supra-sacral lesions $s^{3,4}$ and there are few or no data in the literature regarding the type of ejaculation or sperm parameters ${ }^{3}$ in patients with lesions that include the S2-S3-S4 sacral segments, and who have a flaccid perineum. Knowledge of ejaculation and sperm parameters is important for the provision of appropriate treatment regarding medically assisted procreation for these persons.

The primary aim of this study was therefore to evaluate the type of ejaculation in patients with cauda equina (CE) and conus medullaris (CM) lesions. The secondary aim was to evaluate the quality of the sperm collected.

\section{MATERIALS AND METHODS}

This was a retrospective study carried out between August 1984 and February 2013 in the neuro-urology and genito-sexology laboratory of the
Bouffard-Vercelli Rehabilitation Centre, Cerbère, France and in the Center for the Study and Conservation of Ova and Sperm (CECOS) of the Paule De Viguier Hospital, Toulouse, France.

\section{Patients}

Patients were included if they had lesions of all sacral segments, had passed the phase of spinal shock (6 months post lesion) and complained of anejaculation. Exclusion criteria included repeated uni- or bilateral genito-urinary infections, or sperm expulsion during masturbation. Prior to inclusion, each patient underwent a neurological (to confirm damage of the sacral segments, and to determine the lesion level) and a neuro-perineal examination (to verify: flaccid perineum, no sacral reflexes or voluntary sacral movement, and altered or absent sacral sensation). The severity of the spinal lesions was defined according to the American Spinal Cord Injury Association (ASIA) classification. ${ }^{5}$

\section{Procedures}

All patients underwent vibromassage (VM) to attempt to induce ejaculation. The vibromassage machine (Ferticare, Multicept, Albertslund, Denmark) was applied to the penile fraenulum at a frequency of $100 \mathrm{~Hz}$ and an amplitude of $2.5 \mathrm{~mm}$ for $1-3 \mathrm{~min}^{3}$

Prior to each trial, patients were given oral hydration (VICHY celestins, Allier, France) to make the urine more alkaline, and $40 \mathrm{ml}$ of Ferticult (Fertipro $\mathrm{NV}$, Beernem, Belgium) was instilled into the bladder using a $12 \mathrm{Ch}$ catheter (Wellspect Healthcare, Mölndal-Suède, Sweden). Each antegrade ejaculation

${ }^{1}$ Laboratoire de neuro-urologie et de sexologie, Centre Bouffard-Vercelli, Cerbère, France; ${ }^{2}$ Médecine de la reproduction, Hôpital Paule de Viguier, Toulouse, France and

${ }^{3}$ Département Médullaire, Centre Calvé-Fondation Hopale, Berck-Sur-Mer, France

Correspondence: Dr N Hadiji, Laboratoire de neuro-urologie et de sexologie, Centre Bouffard-Vercelli, Cerbère 66290, France.

E-mail: naderhadiji@yahoo.fr

Received 13 May 2016; revised 26 November 2016; accepted 21 December 2016; published online 14 February 2017 
Table 1 Patients who ejaculated on the first VM trial or who underwent subsequent trials

\begin{tabular}{lccc}
\hline & First VM trial $(\mathrm{n}=11)$ & Subsequent VM trials $(\mathrm{n}=5)$ & VM+midodrine $(\mathrm{n}=26)$ \\
\hline Type of ejaculation & $4 \mathrm{AE}, 6 \mathrm{RE}, 1 \mathrm{ARE}$ & $1 \mathrm{RE}, 1 \mathrm{ARE}$ & $2 \mathrm{AE}, 12 \mathrm{RE}, 4 \mathrm{EAR}$ \\
Mean (range) no. of trials per ejaculation & 1.0 & $1.9(1-6)$ & $4.4(1-7)$ \\
Mean dose of midodrine & $\mathrm{NA}$ & $\mathrm{NA}$ & $22.5 \mathrm{mg}(7.5-30)$ \\
\hline
\end{tabular}

Abbreviations: NA, not applicable; VM, vibromassage.

(AE) was collected and sent to the laboratory. Retrograde ejaculation (RE) was systematically sought in urine that was collected with a sterile catherisation procedure.

If there was no $\mathrm{AE}$ or $\mathrm{RE}$, further trials with midodrine were carried out after an interval of 7 days. Midodrine was administered 30 min prior to the trials. The dose was increased by $7.5 \mathrm{mg}$ at each trial, up to a maximum of $30 \mathrm{mg} .{ }^{6}$

The following clinical signs of AE that occur in patients with supra-sacral SCI were also recorded: arterial hypertension, bradycardia, contractions of the abdominal, lower limb and perineal muscles. These signs were evaluated at the time of $\mathrm{AE}$, or following $3 \mathrm{~min}$ of $\mathrm{VM}$ with no $\mathrm{AE}$.

\section{Sperm parameters}

Sperms were analysed at the CECOS, Hôpital Paule De Viguier, Toulouse, according to the WHO recommendations (2010). Sperms were sought in the urine collected by catherisation as follows: $10 \mu \mathrm{l}$ was examined between slide and cover slide. When no sperm was found, the whole amount of urine was centrifuged at $400 \mathrm{~g}(10 \mathrm{~min})$. The pellet was then suspended in 1-2 $\mathrm{ml}$ of sperm washing medium and analysed as for semen to verify the presence or absence of sperm.

For all samples (antegrade (AE) and bladder (RE) fractions), the following parameters were recorded: sperm concentration, percentage of progressive motility (rapidly progressive+slowly progressive) and vitality. In the case of antero-retrograde (ARE), the mean of the urinary and anterograde concentrations was calculated.

\section{Statistical analysis}

Statistical analysis was carried out using Microsoft office Excel software (Richmond, WA, USA). Means and medians were calculated on the quantitative variables (values reported between square brackets are the extremes). Simple and relative (percentages) frequencies were calculated for the qualitative variables. No statistical comparisons were carried out because of the small sample size.

We certify that all applicable institutional and governmental regulations concerning the ethical use of human volunteers were followed during the course of this research.

\section{RESULTS}

One hundred and fifty-three patients fulfilled the inclusion criteria. Of these, 89 wished to evaluate their fertility potential and consented to undergo the tests. Mean age was 37 years (range 17-66). Mean time since onset of the SCI was 76.6 months (range 10-355). All patients had a flaccid perineum, a complete loss of perineal reflexes and complete loss of voluntary perineal movement. Eighty-five patients had a complete loss of tactile sensation and four had reduced tactile sensation.

The patients were classified into four groups according to the initial lesion level.

Group $1(n=43)$ : T10 (T10-S5) lesion

Group 2 ( $n=27)$ : T11-T12 or L1-L2 (T11-S5) lesion

Group 3 ( $n=14)$ : L3-L4-L5 (L3-S5) lesion

Group $4(n=5)$ : lesion only of S2-S3-S4 (S1-S5)

\section{Type of ejaculation}

The first VM trial was carried out without midodrine (Table 1). Forty-seven patients (52.8\%) did not ejaculate at all (AE, RE or ARE) but did not wish to continue the trials. Eleven (12.3\%) ejaculated during the first VM trial: four AE, six RE and one ARE (Table 1) and did not undergo further trials. Thirty-one (34.8\%) did not ejaculate during the first trial. Of these, 5 patients did not wish to take midodrine and continued trials of VM alone, 2 of whom ejaculated, and 26 underwent trials of $\mathrm{VM}+$ midodrine, 18 of whom ejaculated. The mean number of trials per patient was 4.4 , and the mean dose of midodrine was $22.5 \mathrm{mg}$.

There were no changes in blood pressure or heart rate, and there were no contractions of the lower limbs or perineum in any patient at the time of ejaculation or at the end of a trial in which there was no ejaculation.

\section{Lesion level and ejaculation}

The sperm parameters of 5 of the 31 patients who ejaculated were not found. The remaining 26 patients made up $40 \%$ of Group 1, 35\% of Group 2, 20\% of Group 3 and 25\% of Group 4 (Figure 1).

\section{Sperm parameters}

The semen specimens of 26 of the 31 patients who ejaculated were interpretable. One, or several, ejaculates were obtained for each patient. The mean number per patient was $1.9 .^{1-7}$ Fifty-three ejaculates were obtained from the 234 trials of $\mathrm{VM}$ alone and $\mathrm{VM} \pm$ midodrine (4.4 trials per ejaculation), with a success rate of $22.6 \%$.

Sperm concentration was evaluated in the 53 specimens. The percentage of motile sperm was evaluated in 38 specimens (motility could not be evaluated in the 15 remaining specimens because the samples were too small). Vitality was also evaluated in 12 of the 38 specimens. The percentage of live sperm was evaluated in 23 specimens (it could not be evaluated in the 30 remaining specimens). Of these 23 , motility was also evaluated in 8 .

Sperm concentration was normal in 5 of the 53 specimens (two RE and three ARE), with a mean concentration of $71.7 \times 10^{6} \mathrm{ml}^{-1}$ $\left(34.3 \times 10^{6}-126 \times 10^{6} \mathrm{ml}^{-1}\right)$. The mean percentage of motile sperm was $12.3 \%$ (2-30) (4/5 specimens). The percentage of live sperm was not evaluated in these specimens (Table 2).

Sperm concentration was reduced in 48 of the $53(90.6 \%)$ specimens $\left(<15 \times 10^{6} \mathrm{ml}^{-1}\right)$ with a mean count of $3.5 \times 10^{6} \mathrm{ml}^{-1}$ $\left(0.002 \times 10^{6}-8.1 \times 10^{6} \mathrm{ml}^{-1}\right)$ for $36 \mathrm{RE}, 5 \mathrm{AE}$ and $7 \mathrm{ARE}$ (Table 2).

Vitality was evaluated in 23 specimens obtained from 8 patients. Vitality was $0 \%$ in 15 specimens (65.2\%), with no motile sperm. Mean vitality of the remaining eight specimens (5/8 patients) was $30.1 \%(5-80)$. The mean percentage of motile sperm in seven of these eight specimens was $13.4 \%$ (1-25).

Sperm motility was evaluated in 38 specimens (obtained from 12 patients) in which the mean sperm concentration was $13 \times 10^{6} \mathrm{ml}^{-1}\left(0.2 \times 10^{6}-126 \times 10^{6} \mathrm{ml}^{-1}\right)$. Progressive motility was 


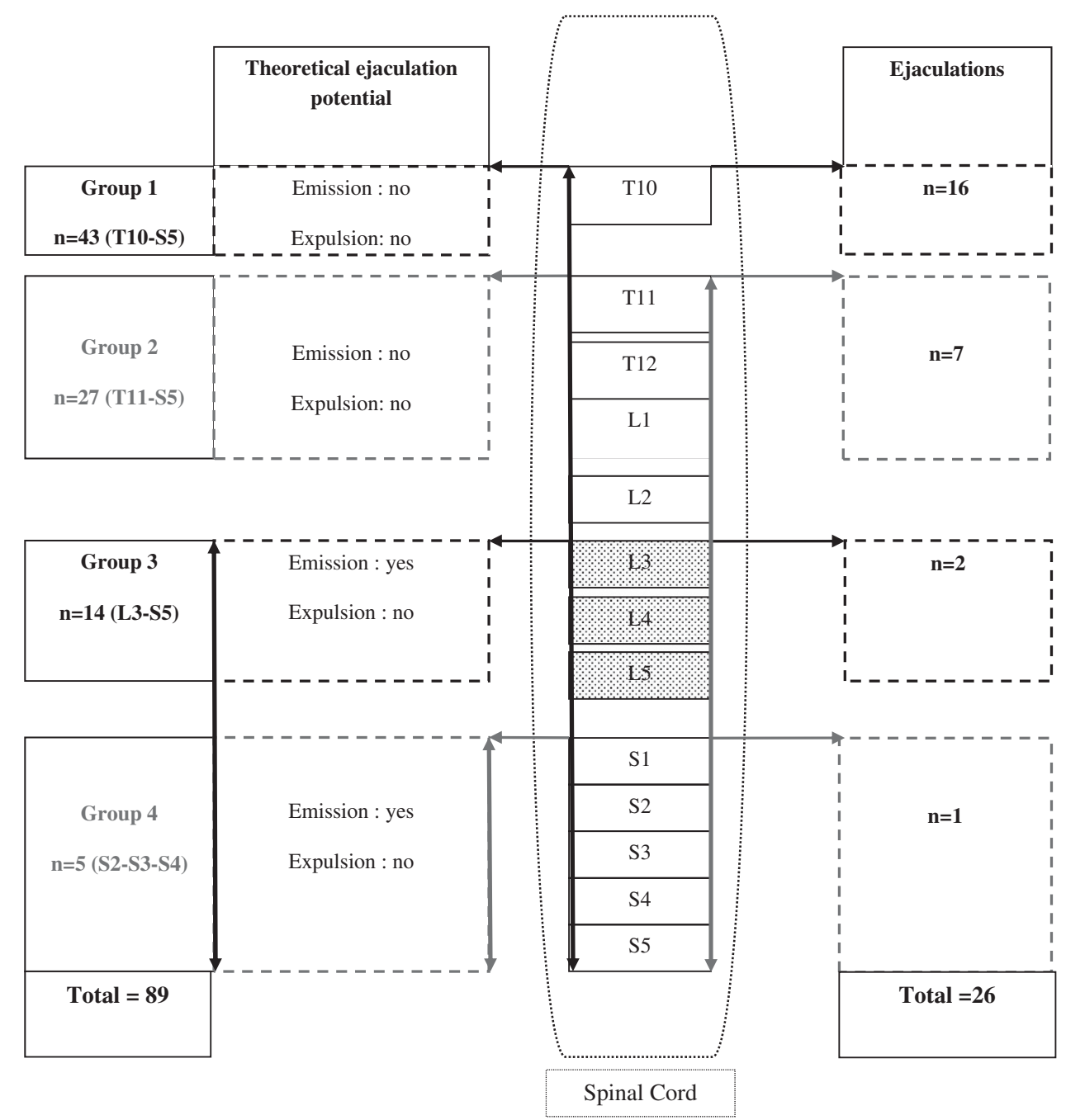

Figure 1 Number of patients who ejaculated as a function of lesion level. Vertical arrows indicate extent of lesion. Horizontal arrows indicate upper limit of lesion. Dotted box indicates Central Spinal Ejaculation Generator. ${ }^{12,15}$ A full color version of this figure is available at the Spinal Cord journal online.

Table 2 Mean sperm concentration in 53 ejaculates obtained from 26 patients

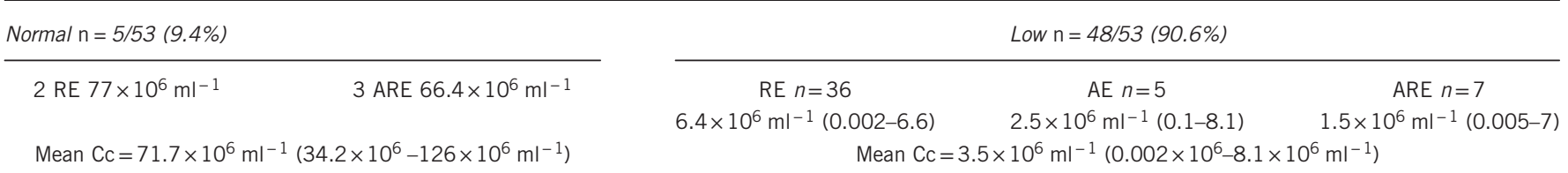

Abbreviations: AE, antegrade ejaculation; ARE, antero-retrograde ejaculation; Cc, concentration; $n$, number of ejaculations; RE, retrograde ejaculation.

Values are means (ranges).

$<32 \%$ (low sperm motility) in 36 of the 38 specimens $(94.7 \%$ ). Motility was $0 \%$ in $18 / 38$ specimens (47.4\%) (5/12 patients). These 18 specimens were from $2 \mathrm{AE}, 3 \mathrm{ARE}$ and $13 \mathrm{RE}$. The other $18 / 36$ specimens $(47.4 \%)$ had a mean progressive motility of $12.9 \%(2-25)$ with a mean sperm concentration of $19 \times 10^{6} \mathrm{ml}^{-1}$ $\left(0.2 \times 10^{6}-126 \times 10^{6} \mathrm{ml}^{-1}\right)$. These 18 specimens (5/12 patients) were from 1 ARE and 17 RE. Mean vitality was $28 \%$ (2-80) (7/18 specimens) (Table 3$)$.

Progressive motility was $>32 \%(35-90)$ in two of the $38(5.3 \%)$ specimens, with a mean sperm concentration of $0.23 \times 10^{6} \mathrm{ml}^{-1}$ $\left(0.07 \times 10^{6}-0.4 \times 10^{6} \mathrm{ml}^{-1}\right)$.
Twenty-eight specimens (7/26 patients, 26.9\%) were of sufficient quality for medically assisted procreation (Table 3 ).

\section{DISCUSSION}

The results of this study showed that it was more difficult to induce ejaculation either with VM alone (13/89) or with midodrine (18/26) in patients with flaccid SCI than in those with supra-sacral lesions ${ }^{3,4}$ (high doses of midodrine were required, and many trials). Sperm expulsion was mostly retrograde (61.3\%). Sperm concentration was nearly always low (90.5\%). Complete necrospermia was found in $65 \%$ of patients and live sperm were asthenic in $95 \%$ of cases. 
Table 3 Sperm motility in 12 patients (38 ejaculates)

\begin{tabular}{|c|c|c|c|c|}
\hline Non-motile sperm & \multicolumn{2}{|c|}{ Low motility $12.9 \%$ [2-25] } & Normal motility ( & 2\%) 47.5\% [35-90] \\
\hline 5 /12 patients (18 ejaculates) & \multicolumn{2}{|c|}{5 /12 patients (18 ejaculates) } & 2 patient & ejaculates) \\
\hline Vitality unknown & Vitality = 28\% (7 ejaculates) & Vitality unknown (11 ejaculates) & Vitality $=50 \%$ ( 1 ejaculate $)$ & Vitality unknown (1 ejaculate) \\
\hline Sperm concentration unknown & \multicolumn{2}{|c|}{ Sperm Cc $=19 \times 10^{6} \mathrm{ml}^{-1}$} & Sperm $\mathrm{Cc}=0.23 \times$ & $6 \mathrm{ml}^{-1}$ (severely low) \\
\hline MAP not possible & \multicolumn{4}{|c|}{ MAP possible (7 patients) } \\
\hline
\end{tabular}

Abbreviations: Cc, concentration; MAP, medically assisted procreation.

Ranges are provided in square brackets.

Lesions of the CE and CM following spinal trauma lead to severe changes in sexual function. ${ }^{7}$ In this particular population, the collection and freezing of sperm should be systematically considered. Medically assisted procreation is likely inevitable, and it is not known whether the loss of sperm viability progresses with time in the case of CM lesions.

Ejaculation is controlled by neurological mechanisms. ${ }^{8,9}$ The thoraco-lumbar sympathetic system (T11-L2) induces sperm emission and closing of the bladder neck (limiting the risk of RE). This centre is under descending inhibitory and/or excitatory control from cortical and sub-cortical structures (thalamic nuclei) that can psychogenically activate or inhibit sperm emission. ${ }^{10,11}$ The parasympathetic system (S2-S3-S4) ensures the glandular secretion of both seminal vesicles and the prostate gland. It participates in the synthesis of seminal plasma.

The sacral somatic system (S2-S3-S4) integrates sensory afferents and generates clonic contractions (bulbo- and ischio-cavernous muscles) for sperm expulsion (ejaculation) via the pudendal nerve. These different centres are coordinated by multiple spinal and supraspinal connections. A central spinal ejaculation generator has been found in animals. ${ }^{9,12}$ This centre integrates somatic sensory peripheral and cortical afferents. It is inhibited by an intraspinal system of inhibitory neurotransmitters. ${ }^{13,14}$

\section{Ejaculation in patients with SCI}

Supra-sacral spinal lesions in which the thoraco-lumbar centres (T11-L2) are spared are no longer under supraspinal control; however, reflex ejaculation remains possible. ${ }^{15}$ If the lesion is around S2-S3-S4, clonic sperm expulsion is theoretically abolished. Sperm emission is conserved if the thoraco-lumbar sympathetic centres are intact (T11-L2). In this case, expulsion is 'dribbling' and never clonic. If T12-L1 is affected, the Adamkiewicz artery may be injured. This artery irrigates the sacral, lumbar and thoracic spinal cord up to T5-T6. ${ }^{16}$ Vascular compensation is rare at this spinal level, which explains why SCI around T12-L1-L2 may result in isolated sacral cord signs, lumbar-sacral cord signs or even thoracic cord signs (Figure 1).

According to the literature, the rate of ejaculation induced by masturbation and/or sexual intercourse is low in patients with SCI (10-15\%). ${ }^{4}$ The threshold of the ejaculatory reflex is much higher in patients with SCI than in healthy subjects because of the loss of sensation, below-lesion motor activity and the loss of supraspinal control. More intense stimulation is required and VM is the first option. ${ }^{8,17} \mathrm{VM}$ activates sacral afferent tracts via the penile dorsal nerve and the sacral spinal centres. ${ }^{18}$ Sectioning or anaesthesia of the penile dorsal nerve has been shown to abolish ejaculation by VM in monkeys. ${ }^{19}$ Sympathetic fibres from T10-L2, which connect to cerebro-spinal sacral tracts, also innervate the penis. ${ }^{9}$ This likely explains why VM can induce sperm emission or dribbling ejaculation in patients who have complete lesions of the sacral cord.
For patients who do not respond to VM alone, administration of midodrine with VM optimises the possibility of ejaculation. ${ }^{17,20}$ Midodrine is a peripheral alpha-mimetic drug which stimulates the sympathetic tracts and the smooth muscle of the internal genetic organs (epididymis, vas deferent, seminal vesicle and prostate) that are primarily innervated by the adrenergic system. Seventy percent of the patients in this study who underwent $\mathrm{VM}+$ midodrine, ejaculated. Ejaculation was mostly retrograde $(61.3 \%)$ because of sympathetic denervation of the bladder neck, and sperm emission was dribbling and was not accompanied by clonic contractions of the perineal muscles (bulbo- and ischio-cavernosus). These results highlight that it is difficult to trigger anterograde ejaculation in patients with SCI who have a flaccid perineum, even with midodrine, corroborating results in the literature. ${ }^{6,21}$ In this specific population of patients with SCI, the rate of ejaculation by VM varies from 11.5 to $35 \%, 3,4,21$ whereas in patients with supra-sacral SCI, the rate is $50-90 \%{ }^{3,4}$ This demonstrates the importance of systematically seeking sperm in the urine in the case of antegrade anejaculation with $\mathrm{VM} \pm$ midodrine (following alkalinisation and Ferticult). Trials should also be repeated with increasing doses of midodrine as most patients who underwent repeated trials with midodrine ejaculated in the present study.

Some authors have suggested that the possibility of ejaculation using VM depends on the lesion level. The chance of ejaculation appears to be greater following cervical lesions than thoraco-lumbar lesions. ${ }^{4}$ Recently, Chéhensse et al. ${ }^{15}$ found that the chance of ejaculation using VM was greater if the L3-L4-L5 myotome were intact. There is a centre for the coordination of ejaculation within these three levels. ${ }^{15}$ Ertekin et al. ${ }^{22}$ found reflex electromyographic (EMG) activity of the cremaster muscle in response to sensory afferent stimulation of S2-S3S4 in patients with lesions above T9. They attributed this to ascending lumbar-sacral afferent circuits, which help to understand the electrophysiological mechanisms of ejaculation in man. These segmental circuits control each other, and their existence explains why the ejaculation reflex is triggered by stimulation of the dorsal penile nerve with VM, in patients with SCI. Lesions of these inter-segmental, sympathetic and parasympathetic circuits could alter the control of ejaculation.

In the present study, 60 patients had lesions which encompassed T11-L1 (Group 1 and Group 2, Figure 1). A lesion of these levels theoretically abolishes both phases of ejaculation. Despite this, 23 patients (38.3\%) ejaculated with VM. Ten patients (Group 3) had lesions which encompassed L3-L4-L5; thus theoretically emission was possible. However, only two (20\%) ejaculated with VM. This low rate of ejaculation in patients with lesions of L3-L4-L5 supports the hypothesis of a central spinal generator for ejaculation at these levels, as suggested by Guiliano et al. ${ }^{9}$ in rats and by Chéhensse et al. ${ }^{15}$ in patients with SCI.

The difference between the cerebro-spinal lesion level (somatic) and the autonomic lesion level could explain the discordances between the theory and clinical observations. 


\section{Sperm parameters}

Most studies of sperm quality have focused on supra-sacral lesions. They have found normal sperm counts ${ }^{3,4,23}$ and alterations in vitality and motility ${ }^{1}$ which do not worsen with time. ${ }^{2}$ Pregnancies can also be initiated at home by intravaginal insemination in these patients. ${ }^{8,23}$ To our knowledge, there are no studies of sperm vitality, motility or concentration in patients with CE.

Moreover, it has been demonstrated in patients with supra-sacral SCI that the deterioration of these parameters is independent of age, time since lesion, whether the lesion is complete or incomplete, the frequency of ejaculation ${ }^{3}$ and testicular hyperthermia. In the present study, all patients were beyond the acute phase of spinal shock and none had experienced ejaculation prior to the trials.

One study found that the number and percentage of motile sperm was greater following cervical than thoracic SCI, with a much lower quality sperm in lesions below T6 (reduction in number and percentage of motile sperm). ${ }^{3}$ The involvement of the accessory glands (epididymis, seminal vesicles and prostate) in the reduction of sperm quality following SCI is not clear. The innervation of the smooth muscle of these glands is under sympathetic control T11-L2), ${ }^{9}$ and it has been shown in animal studies that sperm motility is reduced following ablation of the sympathetic nervous system. ${ }^{24}$

Lesions of the parasympathetic system, which stimulates the glandular secretion of the seminal vesicles and the prostate, could affect the quality of seminal plasma. To our knowledge, there are no studies of seminal plasma in patients with SCI. However, it has been shown that the seminal plasma of patients with supra-sacral SCI reduces the motility and vitality of sperm from healthy subjects. ${ }^{25}$ Changes in epididymal maturation (T11 myotome) could participate in the loss of motility and vitality, as has been reported in one case. ${ }^{26}$ In the present study, seminal plasma markers could not be analysed because of the small numbers of AE. It should be noted that the semen parameters of patients with retrograde ejaculation (RE) could be altered by urine and the manipulation of the semen.

Hormone levels (follicle-stimulating hormone, luteinising hormone, testosterone, inhibin B) have been found to be normal in the majority of patients with SCI above T5 $(82 \%) .^{3}$ In these studies, the T10 myotome (somatic and autonomic innervation of the testicles) was not consistently injured. Hormonal evaluations were not carried out in the present study, but no patient presented with clinical signs of post-injury testosterone deficiency. However, the severely low sperm concentration count found suggests that hormone levels should be evaluated in patients with SCI, as should damage to the autonomic system.

The results of this study suggest that further research should investigate the quality of sperm collected by epididymal aspiration in patients with CE and CM lesions, which has been found to be of better quality than sperm collected using VM in patients with suprasacral SCI.

\section{Limitations}

This study has several limitations: (1) selection of patients based on cerebro-spinal lesion level and not autonomic lesion level (there is a lack of coincidence between somatic and autonomic lesion levels); (2) electrophysiological evaluation of the perineum was not carried out to confirm loss of S2-S3-S4 activity; (3) no evaluation was carried out of psychogenic ejaculation or ejaculation following the least stimulation, as is described in CM lesions, ${ }^{10}$ and which is often dribbling and early, (4) seminal plasma markers were not analysed and ejaculation volume was not reported in all cases; and (5) hormone assessments were not carried out systematically.

\section{CONCLUSION}

The results of this study showed that induced ejaculation following injury of the CE and CM was more difficult to obtain than in patients with supra-sacral lesions, and it was never clonic (because of damage to the sacral centres). High doses of midodrine often needed to be combined with VM to induce ejaculation. The type of ejaculation was variable, anterograde, retrograde or mixed. When midodrine was used, ejaculation was most often retrograde. Analysis of the samples showed a high percentage of low sperm counts and total necrospermia, as well as almost constant asthenospermia. These alterations in sperm parameters suggest that medically assisted procreation is the primary option for these patients should they wish to father children, and cryopreservation of sperm should be systematic.

\section{DATA ARCHIVING}

There were no data to deposit.

\section{CONFLICT OF INTEREST}

The authors declare no conflict of interest.

\section{ACKNOWLEDGEMENTS}

We wish to thank Johanna Robertson for revision of the English of this article.

1 Mallidis C, Lim TC, Hill ST, Skinner DJ, Brown DJ, Johnston WL et al. Collection of semen from men in acute phase of spinal cord injury. Lancet 1994; 343: 1072-1073.

2 Iremashvili V, Brackett NL, Ibrahim E, Aballa TC, Lynne CM. Semen quality remains stable during the chronic phase of spinal cord injury: a Longitudinal Study. J Urol 2010; 184: 2073-2077.

3 Sonksen J. Assisted ejaculation and semen characteristics in spinal cord injured males. Scand J Urol Nephrol Suppl 2003; 213: 1-31.

4 Brackett NL, Ibrahim E, Iremashvili V, Aballa T, Charles ML. Treatment for ejaculatory dysfunction in men with spinal cord injury: an 18-year single center experience. J Urol 2010; 183: 2304-2308.

5 Ditunno JF, Young W, Donovan W, Creasey G. The international standards booklet for neurological and functional classification of spinal cord injury. Paraplegia 1994; 32: 70-80.

6 Soler JM, Prévinaire JG, Plante P, Chartier-Kastler E. Midodrine improves ejaculation in spinal cord injured men. J Urol 2007; 178: 2082-2086.

7 Maynard FM, Bracken MB, Creasey G, Ditunno JF, Donovan WH, Ducker TB et al. International standards for neurological and functional classification of spinal cord injury. Spinal Cord 1997; 35: 266-274.

8 Fin BS, Jens S. Sexual function in spinal cord lesioned men. Spinal Cord 2001; 39: 455-470.

9 Giuliano F, Mac Kenna K, Droupy S, Rampin O, Izard V, Benoit G et al. Vers une approche physiopathologique des troubles de l'éjaculation. Andrologie 1999; 9: 226-246.

10 Courtois F, Charvier K. Premature ejaculation associated with lumbosacral lesions. Spinal Cord 2014; 52: p905-p910 6p.3 Charts.

11 Courtois FJ, Goulet MC, Charvier KF, Leriche A. Posttraumatic erectile potential of spinal cord injured men: how physiologic recordings supplement subjective reports. Arch Phys Med Rehabil 1999; 80: 1268-1272.

12 Truitt WA, Coolen LM. Identification of a potential ejaculation generator in spinal cord. Science 2002; 297: 1566-1569.

13 Carro-Juárez M, Rodríguez-Manzo G. The spinal pattern generator for ejaculation. Brain Res Rev 2008; 58: 106-120.

14 Carro-Juárez M, Rodríguez-Manzo G. Participation of endogenous opioids in the inhibition of the spinal generator for ejaculation in rats. J Sex Med 2009; 6: 3045-3055.

15 Chéhensse C, Bahrami S, Denys P, Clément P, Bernabé J, Giuliano F. The spinal control of ejaculation revisited: a systematic review and meta-analysis of anejaculation in spinal cord injured patients. Hum Reprod Update 2013; 19: 507-526.

16 Lazorthes Y. Guy lazorthes. Surg Neurol 1985; 23: 96-97.

17 Soler JM. Point de vue sur la prise en charge des troubles de l'éjaculation du blessé médullaire. Andrologie 2005; 15: 357-358.

18 Bird VG, Brackett NL, Lynne CM, Aballa TC, Ferrell SM. Reflexes and somatic responses as predictors of ejaculation by penile vibratory stimulation in men with spinal cord injury. Spinal Cord 2001; 39: 514-519.

19 Wieder JA, Brackett NL, Lynne CM, Green JT, Aballa TC. Anaesthetic block of the dorsal penile nerve inhibits vibratory-induced ejaculation in men with spinal cord injuries. Urology 2000; 5: 915-917.

20 Prévinaire JG, Lecourt G, Stoquart G, Soler JM, Plante P. Le recueil de sperme chez le blessé médullaire. Andrologie 2008; 18: 64-69. 
21 Hadiji N, Benbouzid R, Previnaire JG, Leblond C, Mieusset R, Enjalbert M et al. Evaluation du traitement des dysfonctions érectiles et éjaculatoires dans une série de 90 blessés médullaires. Prog Urol 2013; 23: 1489-1493.

22 Ertekin C, Uysal H, Bademkiram F, Baris A. Sacrolumbar intersegmental reflex circuit in men and its relation to the ejaculatory process. Clin Neurophysiol 2007; 118 : 2368-2374.

23 Sonksen J, Fode M, Lochner-Ernst D, Ohl DA. Vibratory ejaculation in 140 spinal cord injured men and home insemination of their partners. Spinal Cord 2012; 50: 63-66.
24 Billups KL, Tillman SL, Chang TS. Reduction of epididymal sperm motility after ablation of the inferior mesenteric plexus in the rat. Fertil Steril 1990; 53: 1076-1082.

25 Brackett NL, Davi RC, Padron OF, Lynne CM. Seminal plasma of spinal cord injured men inhibits sperm motility of normal men. J Urol 1996; 155: 1632-1635.

26 Buch JP. Greatly improved sperm motility from vas deferens sperm retrieval: a case for accessory gland related subfertility in a spinal cord injured man. Paraplegia 1994; 32: 501-504. 